\title{
Safety Assessment of Advanced Imaging Sequences I: Measurements
}

Jensen, Jørgen Arendt; Rasmussen, Morten Fischer; Pihl, Michael Johannes; Holbek, Simon; Villagómez Hoyos, Carlos Armando; Bradway, David; Stuart, Matthias Bo; Tomov, Borislav Gueorguiev

Published in:

IEEE Transactions on Ultrasonics, Ferroelectrics and Frequency Control

Link to article, DOI:

10.1109/TUFFC.2015.2502987

Publication date:

2016

Document Version

Peer reviewed version

Link back to DTU Orbit

Citation $(A P A)$ :

Jensen, J. A., Rasmussen, M. F., Pihl, M. J., Holbek, S., Villagómez Hoyos, C. A., Bradway, D., Stuart, M. B., \& Tomov, B. G. (2016). Safety Assessment of Advanced Imaging Sequences I: Measurements. IEEE Transactions on Ultrasonics, Ferroelectrics and Frequency Control, 63(1), 110-119.

https://doi.org/10.1109/TUFFC.2015.2502987

\section{General rights}

Copyright and moral rights for the publications made accessible in the public portal are retained by the authors and/or other copyright owners and it is a condition of accessing publications that users recognise and abide by the legal requirements associated with these rights.

- Users may download and print one copy of any publication from the public portal for the purpose of private study or research.

- You may not further distribute the material or use it for any profit-making activity or commercial gain

- You may freely distribute the URL identifying the publication in the public portal 


\title{
Safety Assessment of Advanced Imaging Sequences I: Measurements
}

\author{
Jørgen Arendt Jensen, IEEE Fellow, Morten Fischer Rasmussen, Michael Johannes Pihl, \\ Simon Holbek, Carlos Armando Villagómez Hoyos, David P. Bradway, \\ Matthias Bo Stuart and Borislav Gueorguiev Tomov \\ Center for Fast Ultrasound Imaging, Department of Electrical Engineering, \\ Technical University of Denmark, DK-2800 Lyngby, Denmark
}

\begin{abstract}
A method for rapid measurement of intensities $\left(I_{\text {spta }}\right)$, mechanical index (MI), and probe surface temperature for any ultrasound scanning sequence is presented. It uses the scanner's sampling capability to give an accurate measurement of the whole imaging sequence for all emissions to yield the true distributions. The method is several orders of magnitude faster than approaches using an oscilloscope, and it also facilitates validating the emitted pressure field and the scanner's emission sequence software. It has been implemented using the experimental SARUS scanner and the Onda AIMS III intensity measurement system (Onda Corporation, Sunnyvale, CA, USA). Four different sequences have been measured: a fixed focus emission, a duplex sequence containing B-mode and flow emissions, a vector flow sequence with B-mode and flow emissions in 17 directions, and finally a synthetic aperture (SA) duplex flow sequence. A BK8820e (BK Medical, Herlev, Denmark) convex array probe is used for the first three sequences and a BK8670 linear array probe for the SA sequence. The method is shown to give the same intensity values within $0.24 \%$ of the AIMS III Soniq 5.0 (Onda Corporation, Sunnyvale, CA, USA) commercial intensity measurement program. The approach can measure and store data for a full imaging sequence in 3.8 to $8.2 \mathrm{~s}$ per spatial position. Based on $I_{s p t a}$, MI, and probe surface temperature, the method gives the ability to determine whether a sequence is within US FDA limits, or alternatively indicate how to scale it to be within limits.
\end{abstract}

\section{INTRODUCTION}

The US FDA stipulates that intensity levels, mechanical index (MI), and transducer heating have to be measured before studies can be conducted on humans [1]. The guidance document by the US FDA explains the method for obtaining US FDA approval including which standard to use for measurements. These are described in the NEMA UD-2 and UD3 documents as well as the IEC 60601-2-37, IEC 62127-1, and IEC 62359 standards [2], [3], [4], [5], [6]. The standards describe in detail how to conduct measurements and derive the necessary indices. These measurements are often a huge task for manufactures to conduct, as MI and especially intensities depend intimately on the scan setup in terms of focusing depth, elements used, apodization, excitation voltages, transducer, and the imaging sequence, which all have to be taken into consideration. It is increasingly also becoming an issue for research groups, where new advanced imaging sequences and schemes are being developed and ultimately have to be tested on human volunteers and patients. These imaging sequences also have to abide by the same rules as the commercial implementations.

Ultrasound sequences often have hundreds of emission each with different properties. Each emission should ideally be mapped out for all space and intensity levels calculated for the given pulse repetition frequency $\left(f_{p r f}\right)$. Modern ultrasound systems can emit with low F-numbers between 1 and 5 . This leads to transmit pressure distributions that changes energy significantly within distances of $1-5$ wavelengths. The sampling grid therefore has to be dense to capture peaks, which results in long measurement times. Modern sequences can also include spherical waves for synthetic aperture imaging [7] or plane waves [8]. This can include several sources spread out over the aperture or angulation of the plane waves, which distributes the emitted energy over a large area. Long duration signals can also be needed to sample, due to employment of coded imaging [9], [10].

Current intensity measurement systems are oscilloscope based and can often only cope with transmission in one direction at a time, as the oscilloscope does not have information about the imaging mode. This would require that the hydrophone sampling device has full knowledge of the imaging sequence and have the right synchronization signals for start of a new frame and start of a new emissions. In e.g. triplex imaging this can be quite complicated as Bmode, color flow map (CFM) and spectral velocity emissions are interleaved. The sequences are sometimes also very long covering several B-mode images in the CFM sequence. This requires careful synchronization and detailed knowledge of the sequence, which changes as a function of imaging depth and focusing. The sequence, thus, has to be split into the individual emissions and then combined again during intensity calculation, if such a synchronization is not available. Measurement of a single response takes 1-2 seconds due to the hydrophone movement and averaging, and covering a large area for many different emissions can therefore take hours or days for all permutations. The procedure has to be performed for all transducers, setups, and imaging modes. This is very time consuming, and the remedy is often to select only the most important emissions in a sequence and then measure them. These measurements are then applied to others emissions in the sequence. This can be error prone, and often a safety factor has to be imposed to guarantee levels below FDA limits. Such an approach can limit the maximum penetration depth, or adversely affect the quality of velocity estimates, due 
to the poorer signal-to-noise ratio from limiting the emitted energy.

In a research environment this can also be difficult to use consistently for advanced synthetic aperture or plane wave emissions, as all transmitted waves contribute significantly to the overall intensity level. A major source of potential error is also that the scanner has to be reconfigured to single emissions rather than running the actual sequence. This is especially a concern in a research environment, where the resources for implementation and validation of such a scheme are limited.

A method for solving these problems is suggested here, which reduces the measurement time and increases assurance of validated results. It is based on two prior conference papers [11], [12], which are combined here. The method is described in Section III and is based on the Track 1 recommendation from the US FDA [1]. Here the spatial-peak-pulse-average intensity and the mechanical index have to be measured according to IEC and NEMA standards [4], [2], [3]. It also has to be ensured that the probe surface temperature is within acceptable IEC limits [4]. The method uses the sampling capabilities of modern scanners.

Today multi-channel sampling systems for experimental use have been developed like the RASMUS [13], ULA-OP [14], and SARUS (Synthetic Aperture Real-time Ultrasound System) systems [15]. All modern commercial scanners also offer internal real-time sampling of signals from the transducer and some have dedicated research interfaces [16], [17], [18], [19]. The basic idea is therefore to use this sampling capability to acquire the hydrophone signal while the actual imaging sequence is run. The hydrophone signal is then fed into one sampling channel and the signal stored for each emission. A major advantage of this approach is that the pressure signal produced by the actual imaging sequence are measured for all emissions. An unmodified sequence is therefore used, which increases confidence that the measured values represent the actual operating condition of the ultrasound system. The acquisition is performed by the scanner and the hydrophone signal is therefore fully in phase with the emission, so averaging of multiple emissions is easy. All emissions are measured simultaneously for the given position and the full intensity map is acquired in the time it takes for a single frame. This yields a very fast measurement approach. Even using a moderate pulse repetition time between emissions, to avoid reverberations and re-reflections in the water tank, the complete measurement can be performed in seconds for several images. An automatic and generic program can then calculate the complete intensity level for the full image. Intensity mapping of the full sequence in 2-D can be conducted in 1 to 2 hours. Automatic reports are made from the measurements to make it less error prone and more accurate than manual approaches. Any ultrasound scanner with the possibility of connecting an external input to a single sampling channel in the scanner can implement the approach.

Scan sequences are often symmetric and concentrated at the center of the image. In such cases the spatial peak will therefore be on the center acoustical axis of the transducer and the levels need only be measured on this axis. It is, thus, possible to eliminate scanning in the lateral dimension. This demands a very precise alignment of the setup as described in Section IV-A to ensure movement in the correct plane and confirmation that the peak is on the center axis. The selection of the region to scan can be made by employing simulations as detailed in the accompanying paper [20], where linear Field II simulations are shown to closely match the measurements presented here.

The approach has been implemented on the SARUS experimental ultrasound scanner using BK 8820e and BK8670 (BK Ultrasound, Herlev, Denmark) transducers and the AIMS III intensity measurement system (Onda Corporation, Sunnyvale, California, USA) as described in Section IV. Four different imaging sequences are investigated. Results are shown in Section V and commented on in Section VI.

\section{FDA InTENSity AND MechaniCAL IndeX CALCULATION}

The regulatory limits are set by the US FDA [1] They regulate derated values, and related standards [2], [3], [6] specify derated values. These are found by employing an attenuation value of $0.3 \mathrm{~dB} /[\mathrm{MHz} \mathrm{cm}]$ on the pulse using the probe's center frequency. It is found from

$$
f_{0}=\frac{f_{1}+f_{2}}{2}
$$

where $f_{1}$ and $f_{2}$ are the frequencies $-3 \mathrm{~dB}$ down from the peak frequency in the measured spectrum.

The instantaneous intensity is derived from the sampled acoustic pressure $p(n, \vec{r})$ measured in a water bath using a calibrated hydrophone by

$$
I_{i}(n, \vec{r})=\frac{p^{2}(n, \vec{r})}{\rho c}=\frac{p^{2}(n, \vec{r})}{Z},
$$

where $Z=\rho c$ is the characteristic acoustic impedance, $n$ is sample number, $\rho$ is the density, $\vec{r}$ is measurement position, and $c$ is speed of sound. The spatial and temporal peak of $I_{i}$, is denoted $I_{\text {sptp }}$. Averaging over time gives the spatial peak temporal averaged intensity:

$$
I_{s p t a}=\frac{1}{T^{\prime}} \sum_{n=1}^{N} I_{i}\left(t, \vec{r}_{\max }\right) \Delta T
$$

where $N$ is the number of samples in the response, $T^{\prime}$ is the period from pulse to pulse, $\Delta T$ is the sampling interval, and $\vec{r}_{\max }$ denotes the position of maximum intensity. The temporal average intensity is:

$$
I_{t a}(\vec{r})=\sum_{n=1}^{N} \frac{p^{2}(n, \vec{r})}{Z} \frac{\Delta T}{T_{p r f}}
$$

where $T_{p r f}$ is the time between pulse emissions. This is valid for using the same emission continuously, but real imaging sequences uses different pulses in different directions. They must be weighted with their relative time duration in the imaging sequence as

$$
I_{t a}(\vec{r})=\frac{1}{M_{I}} \sum_{i=1}^{M_{I}} \sum_{n=1}^{N} \frac{p_{i}^{2}(n, \vec{r})}{Z} \frac{\Delta T}{T_{p r f}(i)}
$$

where $M_{I}$ is the number of emissions in the complete sequence and $i$ is the emission number. at the center frequency. For 
abdominal scanning the US FDA derated $I_{\text {spta.3 }}$ must be below $720 \mathrm{~mW} / \mathrm{cm}^{2}$, and the derating center frequency should be determined for the individual emissions. This gives the allowable energy level for a given scan site.

The maximum peak negative pressure is limited by the Mechanical Index (MI) given by

$$
M I=\frac{\left|\min \left(p_{d}(n, \vec{r})\right) / 10^{6}\right|}{\sqrt{f_{0} / 10^{6}}}
$$

where $f_{0}$ is the center frequency of the measured pulse and $\min \left(p_{d}(n, \vec{r})\right)$ is the derated peak negative pressure. MI must be below 1.9 , when derated by $0.3 \mathrm{~dB} /[\mathrm{MHz} \mathrm{cm}]$.

The heating of the probe surface must also be measured in the US FDA Track 1 procedure, and can also limit the scan sequence. The temperature increase must be measured for the probe in air and attached to a phantom. They should be performed for the actual scan sequence, and an automatic measurement procedure for this is described in Section III-B.

\section{EFFICIENT MEASUREMENTS OF INTENSITIES FOR ADVANCED IMAGING SEQUENCES}

The hydrophone signal is connected to one of the receive channels in the SARUS scanner, and the signal is measured for all emissions in a sequence for a given spatial position. This ensures the minimum amount of (slow) physical movement of the hydrophone and averaging of responses is also easy, as the measurements are fully synchronized with the emissions. The instantaneous intensity is then calculated by weighting each response according to the relative time duration in the imaging sequence as described in (4).

The method also makes it possible to setup the scanner for the required imaging mode, and then run a program on the scanner, which makes the acquisition. This ensures that the actual sequence using the implemented scanner codes is used, and ensures that the measurement is conducted on the actual scanner and run-code. Implementing this can make the FDA measurements fully automatic, with a completely automatic reporting on FDA levels. It requires that the scanner measurement can be calibrated to obtain the same values as a dedicated intensity measurement system.

\section{A. Calibration of measurement method}

Both the Onda and the SARUS systems have to be calibrated. The signal chain is shown in Fig. 1. It consists of an HGL-400 hydrophone, an ATH-2000 attenuator, an AH-2010 preamplifier, and an angle connector connected to a sampling device through a BNC connection. Each of these must be included in the calibration of the sensitivity in $\mathrm{V} / \mathrm{Pa}$. It must be ensured that the bandwidth of the whole signal chain is flat until $8 f_{0}$ or $40 \mathrm{MHz}$, where $f_{0}$ is the center frequency of the probe [4], [1].

The sensitivity of the hydrophone is adjusted by the potential use of the attenuator and the preamplifier. The sensitivity, gain, and capacitance for the Onda hydrophone, attenuator, and amplifier are found from data supplied by the manufacturer.

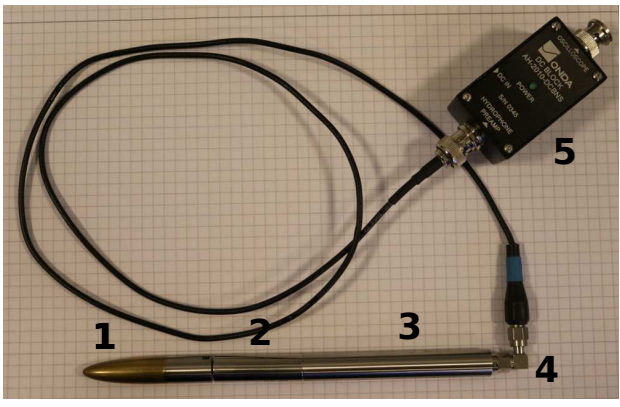

Fig. 1. The signal chain for the hydrophone consists of the HGL-400 hydrophone (1), an ATH-2000 attenuator (2), an AH-2010 preamplifier (3), an angle connector (4), and the power supply (5) connected to a sampling device through a BNC connection.

The sensitivity in $\mathrm{V} / \mathrm{Pa}$ is then calculated based on the user defined center frequency as [21]:

$$
V_{P a}(f)=\frac{10^{\frac{G_{p}(f)}{20}} \cdot 10^{\frac{G_{a}(f)}{20}} M_{c}(f) C_{h}}{\left(C_{h}+C_{a}\right)},
$$

where $G_{a}$ is the attenuator gain in $\mathrm{dB}, G_{p}$ is the preamplifier gain in $\mathrm{dB}, M_{c}$ is the hydrophone sensitivity, $f$ is frequency, $C_{h}$ is hydrophone output capacitance, $C_{a}$ is the attenuator capacitance. If no attenuator is used, the sensitivity is

$$
V_{P a}(f)=\frac{10^{\frac{G_{p}(f)}{20}} M_{c}(f) C_{h}}{\left(C_{h}+C_{p}\right)},
$$

where $C_{p}$ is the preamplifier capacitance. This is used in the Onda measurements with an Agilent DSO5012A oscilloscope and the Soniq 5.0 software [22].

The SARUS system is not designed for calibrated measurements of voltages, and a calibration against the Onda system therefore has to be performed. The basic approach is to measure the same sequence with both systems and then scale the SARUS results from one measurement. Both the Onda software and SARUS system measure a focused single emission sequence, and the scaling factor for the SARUS system is calculated at the spatial peak position of the pressure.

\section{B. Measurement of probe heating}

The heating of the ultrasound probe surface should also be measured during scanning. This includes measuring the probe heating in air and in contact with a suitable phantom. This is easily conducted by attaching a thermocouple to the transducer face and then logging the temperature as a function of time. The temperature rise must at most be 27 degrees in still air and 10 degrees when the probe is attached to a phantom [4]. An automatic setup for such a measurement is described in Section IV-C.

\section{EXPERIMENTAL SETUP}

SARUS is used for emitting the sound and measuring the response for a $3 \mathrm{MHz}$ BK 8820e (BK Ultrasound, Herlev, Denmark) convex array transducer with 192 transducer elements and a BK8670 128-elements linear array probe. An Onda HGL-0400 (golden lipstick) hydrophone is moved by 
the Acoustic Intensity Measurement System AIMS III (Onda, Sunnyvale, California, USA). The hydrophone is connected to an AH-2010 (Onda Corporation, Sunnyvale, USA) $20 \mathrm{~dB}$ amplifier after an ATH-2000 $20 \mathrm{~dB}$ attenuator to prevent overload in the preamplifier.

Four different imaging sequences are used for the experiments. The first sequences focuses a single beam at a depth of $40 \mathrm{~mm}$ on the center axis of the transducer. This is used for the SARUS calibration mentioned above and for comparing the Onda and the SARUS systems. The second duplex scan sequence emits 129 B-mode lines interleaved with 129 flow emissions [23], [24]. The third example is a vector flow imaging scheme (VFI sequence) with 17 emission directions of 32 emissions each followed by a 129 lines B-mode image [25]. The fourth is a duplex Synthetic Aperture (SA) sequence with flow emissions inter-leaved with B-mode emissions suitable for high dynamic range flow imaging [26]. The method is, however, not limited to these modes as any other imaging scheme can be used (synthetic aperture, triplex, plane wave, continuous wave, 3-D, etc.).

To avoid reverberations and multiple reflections in the tank, the pulse repetition frequency can be lowered during measurements. It is possible to use any low pulse repetition frequency, as the measured intensities can be linearly scaled by the one used in clinical measurements to give the correct intensity level. The hydrophone amplifier is connected to one receive channel in SARUS, which has a maximum input range of \pm 1 volts with 12-bits precision. The SARUS and Onda systems are controlled using Matlab (Mathworks, MA, USA) through internally made libraries containing $\mathrm{C}$ and Matlab code to fully automate the measurements. The emission and hydrophone measurement are performed by the same system and are therefore fully in phase. Averaging even complicated measurement sequences is therefore possible without phase errors, and it is, thus, not necessary to resort to phase correction schemes used in other systems for getting usable averages.

The basic setup is shown in Fig. 2 with the mounting of the transducer on the fixed boom and the hydrophone mounted on the translation stage. The coordinate system follows the usual convention of $z$ being the depth direction, $x$ along the transducer crystals, and $y$ in the elevation direction.

\section{A. Transducer alignment}

The hydrophone must be moved relative to the transducer coordinate system, and alignment therefore has to be performed. A single focused field with an F-number of 2 and using 64 elements is employed. This has a well defined peak value, which is found by searching around the focus and at two depths before and after the focus. This gives a number of points in 3-D space from which the unit vector for the transducer coordinate system can be found.

The hydrophone is first moved across the beam in the $x$ direction to find a value before the maximum at $x_{m}-\Delta_{x}$ and two positions after the maximum at $x_{m}$ and $x_{m}+\Delta_{x}$, where $\Delta_{x}$ is the step size. A second order polynomial is then fitted to the energy of the response, and the true peak position is found

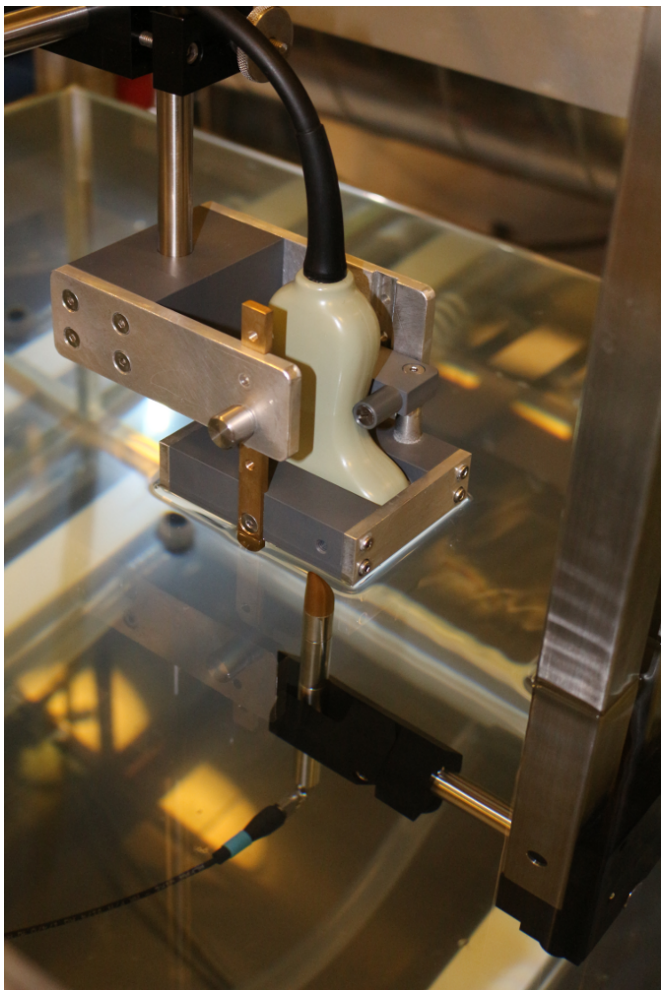

Fig. 2. Mounting of the BK8820e convex array probe in the Onda system. The probe is fixed and the hydrophone below is moved relative to the probe.

by the interpolation formula [27]:

$$
x_{i n t}=x_{m}-\Delta_{x} \frac{E\left(x_{m}+\Delta_{x}\right)-E\left(x_{m}-\Delta_{x}\right)}{2\left(E\left(x_{m}+\Delta_{x}\right)-2 E\left(x_{m}\right)+E\left(x_{m}-\Delta_{x}\right)\right)},
$$

where $E(x)$ is the energy of the pressure response at position $x$. The parametric fitting reduces the number of steps for finding the peak and gives a very accurate determination of the peak position. The procedure is then repeated in the $y$ direction. Making this before and after the focus gives two points from which the unit vector for the transducer coordinate system can be found, and the position of the focus peak gives the distance to the transducer. The hydrophone can then be moved safely in the transducer coordinate system.

\section{B. Measurement protocol}

The basic protocol is:

1) Setup SARUS to transmit a focused field along the acoustical axis of the transducer.

2) Move the transducer manually to obtain the maximum pressure signal on the axis of the transducer.

3) Execute the automatic alignment program to find the center position of the aperture and calculate the unit vector for the acoustical axis.

4) Run the imaging sequence on the scanner to make the setup and store a description of the sequence.

5) Determine the needed measurement area from simulation of the sequence.

6) For each position in the region of interest, measure all emissions in the imaging sequence a number of times, 
and store the averaged data in Matlab files for a given position.

7) Make an automatic report on derated intensities and MI from the acquired data.

In this approach SARUS is programmed in the same way as for conducting a clinical study. After the programming a separate script is run, which modifies the acquisition to measure only the hydrophone signal. The script then runs the clinical emission sequence automatically, and thereby ensures that the correct sequence is executed by the scanner. The hydrophone records the pressure waves for all emissions at one spatial position in the time span for one image. The hydrophone is then moved over the user-specified region of interest and the measurements are conducted. After the acquisition a Matlab script calculates the intensity and MI distributions and generates an HTML page with the results and graphs for the distributions and the calibration.

\section{Setup for probe heating measurement}

The probe surface temperature should also be automatically measured to ensure safe operation. The SARUS system is set-up for the user's scanning sequence, and a temperature measurement script is then run under Matlab. It controls the starting and stopping of the scanner and logs the temperature of the probe, and it automatically shuts down the scanner if the temperature is above a preset limit or if a predetermined time limit is met. An Almemo 2390-5 temperature DAQ device (Ahlborn Mess- und Regelungstechnik GmbH, Holzkirchen Germany) is used with a USB interface to the controlling PC. An Almemo ZA 9020-FS thermocouple is attached to the probe surface during measurement. A large $(20 \times 20 \mathrm{x}$ $20 \mathrm{~cm}$ ) agar-agar based phantom (Danish Phantom Surface no. 657, Frederikssund, Denmark) is couple to the probe through normal clinical scan gel. The phantom has normal acoustic properties with a sound speed of $1544.4 \pm 0.3 \mathrm{~m} / \mathrm{s}$, an attenuation of $0.52 \pm 0.02 \mathrm{~dB} /[\mathrm{MHz} \cdot \mathrm{cm}]$, scattering properties resembling soft tissue, and a density of 1055.0 $\mathrm{kg} / \mathrm{m}^{3}$. The heat capacity of agar is $3900 \mathrm{~J} /[\mathrm{kg} \cdot \mathrm{K}]$ and the thermal conductivity is $0.6 \mathrm{~W} /[\mathrm{m} \cdot \mathrm{K}]$ [28], corresponding to the properties required for a temperature test phantom [4]. A HTML-based report is generated with the measured data, along with an indication whether the sequence obeys US FDA and IEC limits for transducer surface temperature rise.

\section{RESULTS}

This Section shows the results from the measurements. The calibration of the system is shown in Section V-A and the measurement results for the four sequences are shown in Sections V-B to V-E.

\section{A. Calibration of SARUS}

The peak pressure waveforms measured on the acoustical axis of the probe by both systems are shown in Fig. 3 . The SARUS waveform is scaled by a factor of 1.2314 , to have the same energy in both response. Small differences are seen between the waveforms, which are due to bandwidth
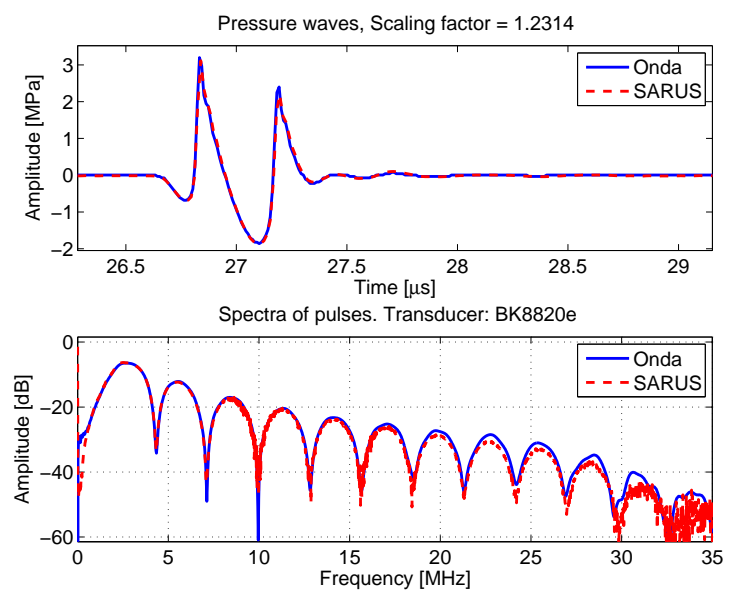

Fig. 3. Calibration waveforms measured at the peak pressure position for a single focus emission with an F-number of 2 . The blue curve is from the Onda system, and the red is from SARUS scaled by a factor 1.2314 to yield the same energy as from the Onda system. The bottom graph shows the corresponding Fourier transforms.

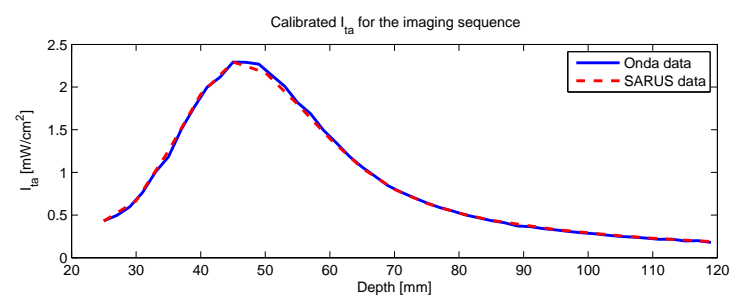

Fig. 4. $I_{t a}$ measured by the SARUS scanner after calibration (red curve) compared to the Onda system values (blue curve) for the single emission sequence.

limitations in the SARUS input stage. This can also be seen in the bottom graph, which displays the Fourier spectrum of the two waves. Until $12 \mathrm{MHz}$ the graphs are similar, and after 15 $\mathrm{MHz}$ the deviation starts to increase due to the SARUS low pass filter. It has a $50 \Omega$ input impedance and a $-3 \mathrm{~dB}$ cutoff frequency of $40.8 \mathrm{MHz}$ to avoid aliasing in the sampling process. Despite this the maximum deviation is $0.74 \mathrm{~dB}$ below frequencies of $15 \mathrm{MHz}$, which is the frequency range for transducers used on SARUS.

The difference between the two measurement systems was investigated by moving the hydrophone for depths from $20 \mathrm{~mm}$ to $120 \mathrm{~mm}$ along the acoustical axis of the field. Fig. 4 shows the resulting $I_{t a}$. The calibrated $I_{\text {spta }}$ is $2.29 \mathrm{~mW} / \mathrm{cm}^{2}$ due to the low $f_{\text {prf }}$ of $100 \mathrm{~Hz}$. The relative error of $0.24 \%$ between the AIMS III system and SARUS indicates a negligible difference between the systems. The single channel sampling in SARUS, thus, yield correct intensity levels.

\section{B. Measurements for single focus emission}

The intensity measured for the single focus sequence is shown in Fig. 5, where the left image shows the derated $I_{t a}$ in $\mathrm{dB}$ relative to $1 \mathrm{~mW} / \mathrm{cm}^{2}$. Colors below red indicates areas that are within the FDA limit of $720 \mathrm{~mW} / \mathrm{cm}^{2}$. The white circle indicates the position of $I_{s p t a}$, and in this case it coincides with 


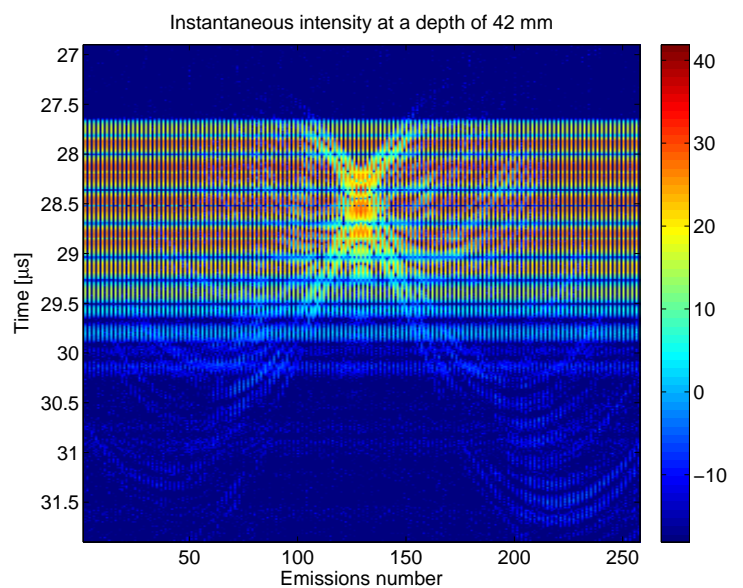

Fig. 6. Instantaneous intensity distribution as a function of time and emission number, when the hydrophone is $42 \mathrm{~mm}$ from the probe. The color values are in $\mathrm{dB}$ relative to $0.1 \mu \mathrm{W} / \mathrm{cm}^{2}$.

the position of the transmit focus. The shape of the emitted field can also be seen with an intensity level that concentrates around the focus and diverges after it. The measurement was conducted at a pulse repetition frequency of $100 \mathrm{~Hz}$ where $I_{\text {spta }}=3.28 \mathrm{~mW} / \mathrm{cm}^{2}$. The pulse repetition frequency can, thus, be scaled by a factor of 219 before reaching the FDA limit.

The right image in Fig. 5 shows the derated MI on a linear scale. Again a red color value indicates values getting above the FDA limit of 1.9. The white circle indicates the position of the maximum value, which here is 0.81 . It also coincides with the transmit focus at a depth of $40 \mathrm{~mm}$. The transmit voltage can be scaled by a factor of 2.35 before reaching the FDA limit, when assuming a linear scaling. This will, however, depend on the transmit amplifier and the nonlinear propagation. A new measurement should therefore be conducted, if a new transmit voltage is used.

\section{Measurements for Duplex flow}

The duplex scan sequence mixes flow and B-mode emissions, and yields a complex energy distribution in the medium. The instantaneous intensity is shown in Fig. 6 as a function of emission number and time. The intensities are shown with a 60 $\mathrm{dB}$ dynamic range in $\mathrm{dB}$ relative to an instantaneous intensity of $0.1 \mu \mathrm{W} / \mathrm{cm}^{2}$ The data are acquired at a depth of $42 \mathrm{~mm}$ with the hydrophone at the center axis of the transducer coinciding with the transmit focus for the B-mode sequence.

The sequence alternates between flow and B-mode emissions and every second emission is a four cycle transmission suitable for velocity estimation. The butterfly shape at the image center is the emitted B-mode field, since the imaging beam is swept across the hydrophone position. To avoid rereflections in the water tank the measurement is conducted at an $f_{p r f}$ of $200 \mathrm{~Hz}$. The real measurement then gives a value of $I_{t a}=245 \mathrm{~mW} / \mathrm{cm}^{2}$ at $f_{p r f}=8 \mathrm{kHz}$.

The spatial distribution of $I_{t a}$ is shown in Fig. 7. The hydrophone has been moved to the different spatial positions indicated and the signals averaged for two emissions. The peak value of $I_{\text {spta }}=14.42 \mathrm{~mW} / \mathrm{cm}^{2}$ is at $66 \mathrm{~mm}$. The pulse

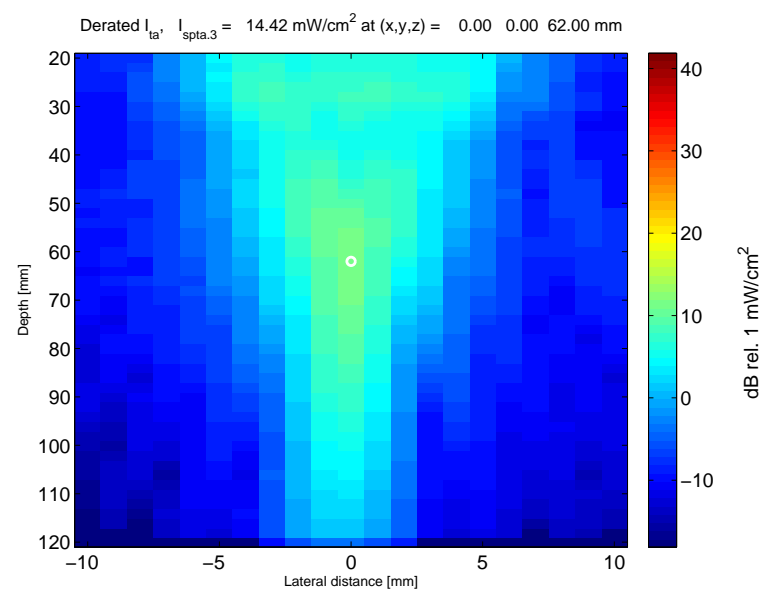

Fig. 7. Distribution of $I_{t a}$ as a function of spatial position for the duplex sequence. The location of the peak intensity $I_{\text {spta }}$ is shown by the blue circle. The color values are in $\mathrm{dB}$ relative to $1 \mathrm{~mW} / \mathrm{cm}^{2}$.

repetition frequency can, thus, be scaled by a factor of nearly 50 and still be below the FDA limit of $I_{\text {spta. }}=720 \mathrm{~mW} / \mathrm{cm}^{2}$. This sequence is, thus, limited to an $f_{p r f}$ below $10 \mathrm{kHz}$. The main intensity contribution is at the center of the image, where the flow emissions are emitted. Away from the acoustic center axis the intensity drops by the distance and attains a level roughly $15-20 \mathrm{~dB}$ below the peak level. This intensity level is due to the B-mode emissions, which are spread over a large imaging region. Here the maximum value is also around 65 $\mathrm{mm}$, due to the transducer's elevation focus.

\section{Measurements for VFI sequence}

The VFI sequence alternates between a B-mode image and vector flow imaging emissions in 17 directions with 32 emissions per direction. The VFI sequence is focused at $105 \mathrm{~mm}$, and the B-mode image is focused at $40 \mathrm{~mm}$. The intensities were scanned for depths from 20 to $120 \mathrm{~mm}$ and from -40 to $+40 \mathrm{~mm}$ in the lateral direction. The measurements were conducted at $f_{p r f}=2 \mathrm{kHz}$. The resulting derated values are shown in Fig. 8.

The intensities are fairly uniformly distributed in the region of the VFI imaging and the peak value of $31.9 \mathrm{~mW} / \mathrm{cm}^{2}$ is found close to the transducer due to the derating of the measured values. The higher values in the VFI region is due to the four cycle pulse used for velocity imaging. The largest derated MI value of 1.32 is found at the focus of the B-mode image, where the F-number is low. The larger region for the flow compared to the duplex sequence decreases the overall intensity, and $f_{p r f}$ can be scaled to a value of $45 \mathrm{kHz}$.

\section{E. Measurements for SA sequence}

The SA sequence was developed for flow imaging, where 7 emissions of spherical waves are spread out over the 128 elements BK 8670 linear array. Every 8th emission is used for B-mode imaging, and this emission is made for all 128 elements. The sequence is, thus, 1024 emissions long and is designed so the 7 flow emissions are repeated continuously. 

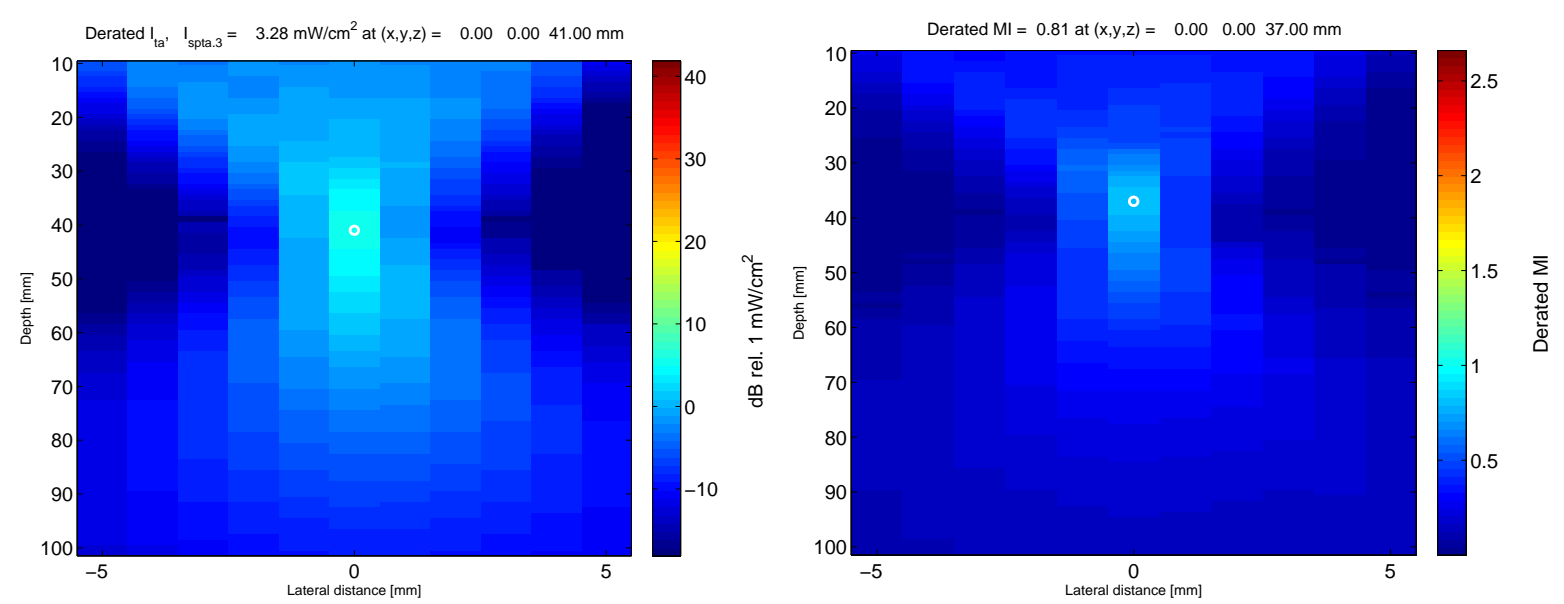

Fig. 5. Results for the single emission sequence as a function of spatial position. The derated temporal averaged intensity is shown in the left graph and the derated MI is shown on the right. The white circles indicate the position of the peak values.
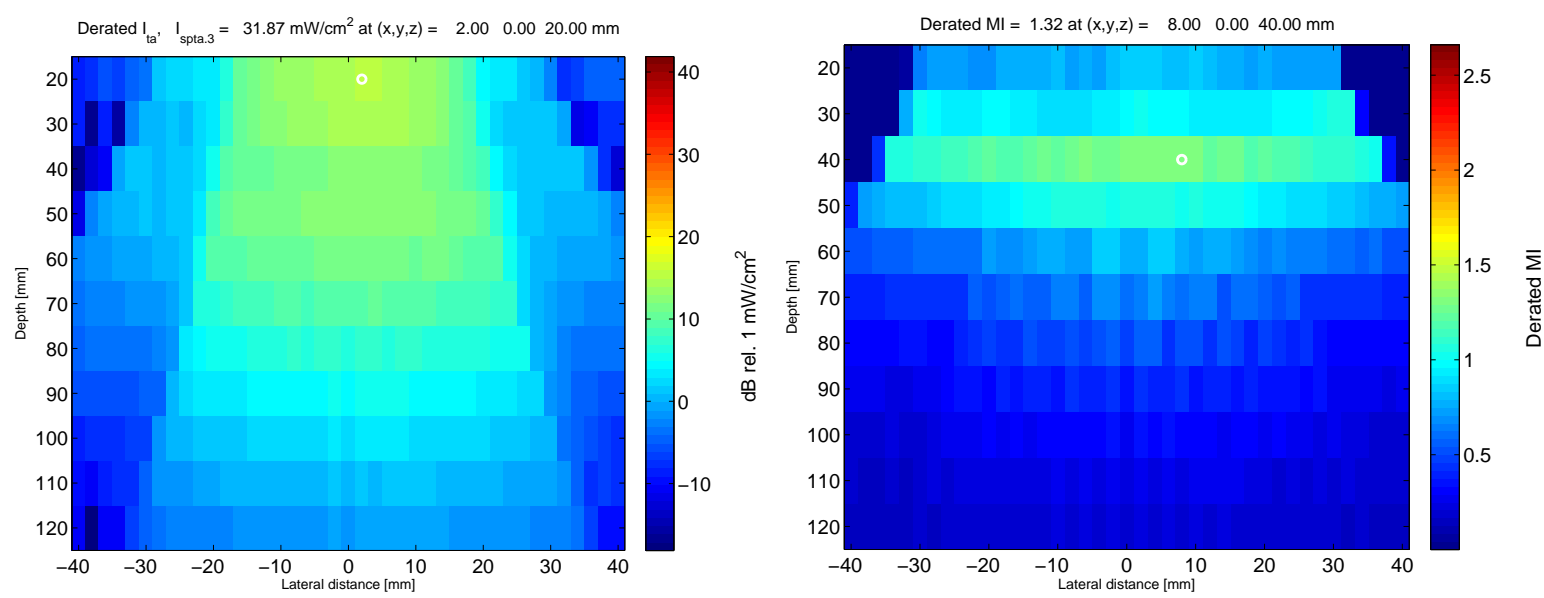

Fig. 8. Results for the vector flow imaging (VFI) sequence as a function of spatial position. The derated temporal averaged intensity is shown in the left graph and the derated MI is shown on the right. The white circle indicates the position of the peak values.

The focus is behind the aperture giving rise to spherical waves, where the intensity rapidly drops after the transducer surface. The intensities were scanned close to the transducer for depths from 10 to $17 \mathrm{~mm}$ and from -5 to $+5 \mathrm{~mm}$ in the lateral direction. The measurements were conducted at $f_{p r f}=500$ $\mathrm{Hz}$. The resulting derated values are shown in Fig. 9.

Here a very uniform and low intensity with a peak value of $4.81 \mathrm{~mW} / \mathrm{cm}^{2}$ is found close to the transducer. MI is 0.91 indicating that both the transmit voltage and $f_{p r f}$ can be increased or coded excitation could be used [29].

\section{F. Measurement of transducer surface temperature rise}

The transducer surface temperature rise for the SA scan sequence is shown in Fig. 10. Here the blue curve is in air and the red curve is when measured on a phantom. The normal exponential curve is typical for such temperature measurements, and it can be seen that the temperature rises from the ambient value of $23.7^{\circ}$ to $47.3^{\circ}$, which is within the acceptable limits of a temperature rise of $27^{\circ}$. For the phantom measurement it is from $24.3^{\circ}$ to $30.0^{\circ}$ after $1 / 2$ hour of heating. Here the limit is a maximum of $10^{\circ}$ rise. Both are, thus, within IEC limits [4].

\section{G. Measurement times}

All the $21 \times 101=2,121$ measurements were acquired in 8,430 seconds for the duplex sequence for both emission, data transfer, storage, and motion of the hydrophone. A single measurement, thus, takes 3.97 seconds including the 2.58 seconds for emitting the 258 emissions at $f_{p r f}=100 \mathrm{~Hz}$. The data reading, storage and movement part therefore takes $1.39 \mathrm{~s}$.

The VFI sequence took $5,389 \mathrm{~s}$ for the $41 \times 13=533$ measurements. Each contains 673 emissions repeated five times for 3,365 emissions at an $f_{p r f}$ of $2 \mathrm{kHz}$. This corresponds to $1.68 \mathrm{~s}$ of emissions and the reading, storage, and movement part therefore takes $8.2 \mathrm{~s}$, as there is more data to load and average.

The single focus sequence contained 1,001 measurements and was conducted in 3,900 seconds for a storage time of $3.8 \mathrm{~s}$ per sequence at each spatial point. 

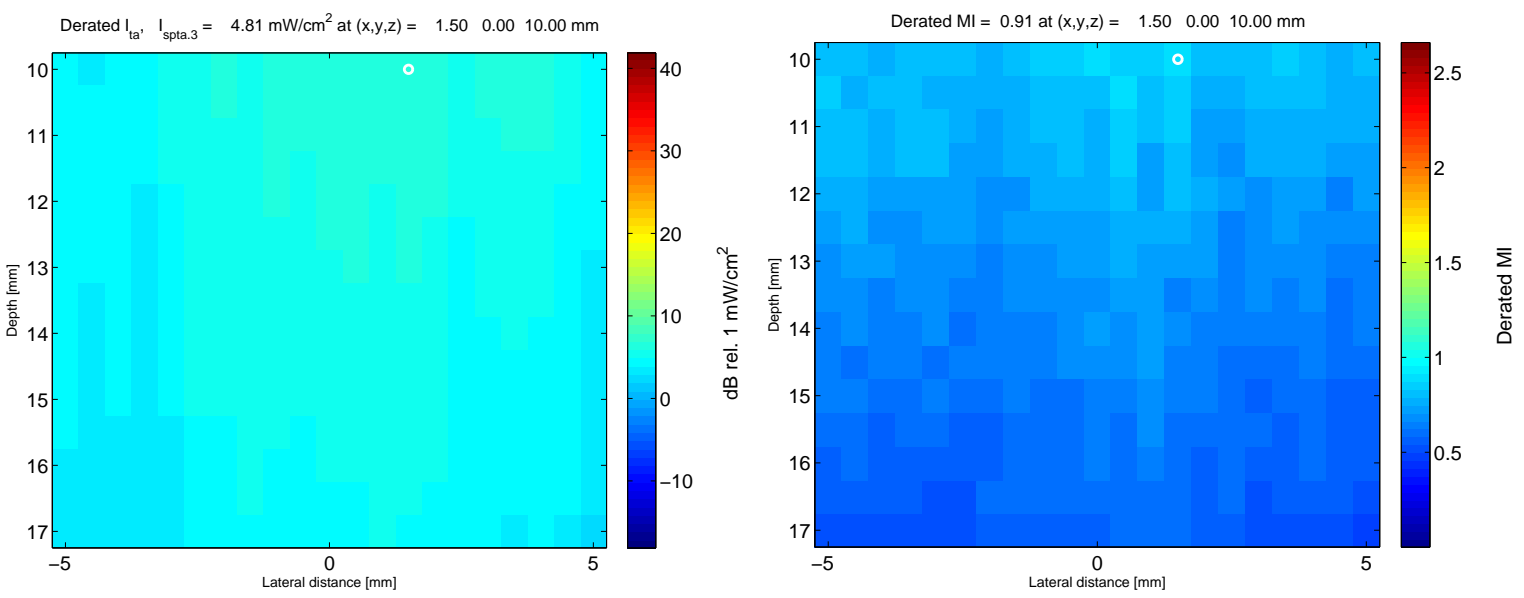

Fig. 9. Results for the synthetic aperture vector flow imaging (SA) sequence as a function of spatial position. The derated temporal averaged intensity is shown in the left graph and the derated MI is shown on the right. The white circle indicates the position of the peak values.

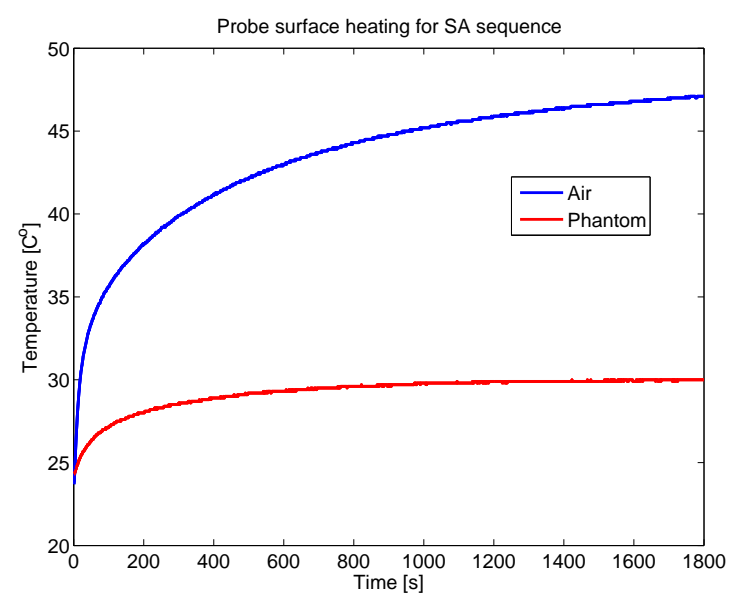

Fig. 10. Temperature rise as a function of time for SA sequence. The blue curve is in air and the red curve is when measured on a phantom for $1 / 2$ hour.

\section{DISCUSSION}

A method for the measurement and simulation of advanced imaging sequences has been presented. The scheme can automatically measure any imaging sequence setup on the scanner as a separate program independent of the actual imaging just has to be run. The method devised here thereby makes it possible to validate the whole setup of the scanner including the real time scan control. The current method for measuring intensity has to break up the sequence into the individual emissions and then after measurements make a calculation of the total intensity and MI. This can be correct, but can also give too conservative estimates, but most importantly such an approach does not guarantee that the actual software in the scanner implements the sequence desired. This can be guaranteed and validated automatically with the presented approach.

The measurement time for one point was $3.97 \mathrm{~s}$ for the duplex sequence. Other experiments have shown that inserting an attenuating mat underneath the transducer at the bottom of the tank can reduce re-reflections sufficiently to not influence the measurement. This makes it possible to increase $f_{p r f}$ to e.g. $2 \mathrm{kHz}$ or more. The emission time is then reduced by a factor of 20 to $0.129 \mathrm{~s}$ per measurement. Currently SARUS has a waiting time between measurements, which could be reduced. Also the read out time for the measurement can be reduced, so that the primary time determining the measurement speed is the movement of the hydrophone. Ideally this can reduce the total time to $0.129+0.139=0.268 \mathrm{~s}$ plus time for the movement, where the $0.139 \mathrm{~s}$ is the storage time. The total measurement time in an optimized set-up could therefore be kept to one second per point for the duplex sequence. The more complicated VFI sequence consists of $129+32 \times 17=673$ emissions. Measuring down to a depth of $15 \mathrm{~cm}$ necessitates a pulse repetition time of $100 \mu \mathrm{s}$ for a full emission time of $0.0673 \mathrm{~s}$. This generates $9.4 \mathrm{Mbytes} / \mathrm{emission}$ using a $70 \mathrm{MHz}$ sampling frequency, when sampling the full response. It is possible for SARUS to store 5.1 Mbytes of data per second to disk per measurement card in the system. This would take 1.8 $\mathrm{s}$ per frame and storage time would dominate the measurement process. This can significantly be reduced by adapting the sampling to the actual depth. For this sequence the largest span in arrival times $10 \mathrm{~mm}$ from the transducer is $20.3 \mu \mathrm{s}$. Reducing the sampling time to $30 \mu$ s would reduce the storage time to $0.54 \mathrm{~s}$, and this makes it possible to maintain the measurement time of $1 \mathrm{~s}$ per point. The largest measurements conducted here span $80 \times 100$ points, and thereby could be optimized to take 8,000 seconds or 2 hours $12 \mathrm{~min}$ for the whole measurement.

The measurement at a reduced $f_{p r f}$ ensures freedom from re-reflections, which can bias the measurements, and the underlying assumption is that intensities directly can be scaled with the emission frequency. This is only valid in a linear regime, and effects from water heating, probe heating, and larger current drains on the output amplifiers might change the results. All of these effects, however, reduce the efficiency of the system, and the intensity is probably lower than the scaling of $f_{p r f}$.

In many sequences the important areas of the peak intensity 
and MI are confined to a smaller region. This can be uncovered using simulations as shown in the accompanying paper [20]. Although a linear simulation model is used, it is possible to predict both derated values for $I_{\text {spta }}$ and MI to a deviation of less than $40 \%$, which is sufficient to predict a narrow region for the peak values and how closely the spatial sampling should be performed. This also makes it possible to rapidly prototype new sequences, and validate the possibility for implementation using simulation by employing a measured impulse response for the probe used.

Often ultrasound sequences are not limited by either intensity level or MI but by the probe surface heating. This is especially true for modern SA and plane wave sequences, which distribute the ultrasound energy over a wider area and do not use focused emissions. Here the probe surface temperature rise is the limiting factor, and an automatic scheme for measuring this has also been developed. The temperature is measured with a thermocouple, and a program is run under Matlab for acquisition and controlling the SARUS scanner. This ensures that an unmodified sequence is used and the test is again generic and independent of the user's program. An automatic web based report is also generated and indicate whether the IEC rules for temperature rise are obeyed.

The developed method is not limited to the US FDA Track 1 approach. The Track 3 approach is based on determining MI and the Thermal Index (TI). This can be estimated from a radiation force balance system or from scanning the pressure signals in a region suitable for calculating the emitted power [3]. The sampling density should be properly determined for the ultrasound field, and new calculation routines should be validated against a reference model. The principles behind the measurements and how to conduct them are, however, similar to Track 1.

Finally it should be noted that there are also alternative ways of measuring the power induced by an ultrasound sequence. This includes thermo acoustic sensors which: "are a very simple and low-cost alternative for the determination of local temporal-averaged intensities. Here, inclusion of all contributing pulses and temporal averaging is done by the sensor inherently without the need for synchronization to individual pulses and pulse sequences. The measurement technique is based on the transformation of the incident ultrasonic energy into heat inside a small-sized cylindrical absorber." (from http://www.ptb.de/cms/en/ptb/fachabteilungen/abt1/fb16/ag-162/thermo-acoustic-sensors.html). This can be a very rapid method for assessing the thermal properties, but does not give a detailed understanding of the emitted pressure and how to scale the imaging sequence by lowering the pulse repetition frequency for reducing intensity and probe heating or lowering the transmit voltage to reduce MI.

\section{CONCLUSIONS}

A method for measuring the complete intensity map, distribution of MI, and probe surface temperature has been suggested and investigated. It uses one scanner input to acquire the pressure data, and it was demonstrated that this is accurate within $0.24 \%$ of the results from the Onda AIMS III intensity measurement system for a single calibration measurement. The approach can also be used to validate the transmitted field and that the scanner is emitting pulses as expected. Based on $I_{\text {spta }}$, MI, and probe temperature it can automatically be determined whether the imaging sequence obeys the US FDA track 1 procedure for ultrasound sequences, and modifications to the sequence can be suggested to make it usable for in-vivo scanning in case it exceeds the limits.

\section{ACKNOWLEDGEMENT}

This work was supported by grant grant 82-2012-4 from the Danish Advanced Technology Foundation and by B-K Ultrasound ApS.

\section{REFERENCES}

[1] FDA, "Information for manufacturers seeking marketing clearance of diagnostic ultrasound systems and transducers," Center for Devices and Radiological Health, United States Food and Drug Administration, Tech. Rep., 2008.

[2] NEMA, "Acoustic output measurement standard for diagnostic ultrasound equipment, revision 3," National Electrical Manufactures Association, Tech. Rep. NEMA-UD2, January 2004.

[3] _ _ "Standard for real-time display of thermal and mechanical acoustic output indices on diagnostic ultrasound equipment, revision 2," National Electrical Manufactures Association, Tech. Rep. NEMA-UD3, January 2004.

[4] IEC, "Medical electrical equipment - part 2-37: Particular requirements for the basic safety and essential performance of ultrasonic medical diagnostic and monitoring equipment," International Electrotechnical Commision, Tech. Rep. IEC 60601-2-37, 2015, edition 2.1 2015-06.

[5] _ - "Measurement and characterization of medical ultrasonic fields up to $40 \mathrm{MHz}$," International Electrotechnical Commision, Tech. Rep. IEC 62127-1, 2013.

[6] — - "Ultrasonics - Field characterization - Test methods for the determination of thermal and mechanical indices related to medical diagnostic ultrasonic fields," International Electrotechnical Commision, Tech. Rep. IEC 62359, 2010, edition 2.1 2015-06.

[7] J. A. Jensen, S. Nikolov, K. L. Gammelmark, and M. H. Pedersen, "Synthetic aperture ultrasound imaging," Ultrasonics, vol. 44, pp. e5e15, 2006

[8] M. Tanter and M. Fink, "Ultrafast imaging in biomedical ultrasound," IEEE Trans. Ultrason., Ferroelec., Freq. Contr., vol. 61, no. 1, pp. 102119, January 2014.

[9] M. O'Donnell, "Coded excitation system for improving the penetration of real-time phased-array imaging systems," IEEE Trans. Ultrason., Ferroelec., Freq. Contr., vol. 39, pp. 341-351, 1992.

[10] T. Misaridis and J. A. Jensen, "Use of modulated excitation signals in ultrasound, Part I: Basic concepts and expected benefits," IEEE Trans. Ultrason., Ferroelec., Freq. Contr., vol. 52, pp. 192-207, 2005.

[11] J. A. Jensen, M. F. Rasmussen, M. B. Stuart, and B. G. Tomov, "Rapid measurements of intensities for safety assessment of advanced imaging sequences," in Proc. SPIE Med. Imag., vol. 9040 90400Z-1, 2014.

[12] — "Simulation and efficient measurements of intensities for complex imaging sequences," in Proc. IEEE Ultrason. Symp., 2014, pp. 11641167.

[13] J. A. Jensen, O. Holm, L. J. Jensen, H. Bendsen, S. I. Nikolov, B. G. Tomov, P. Munk, M. Hansen, K. Salomonsen, J. Hansen, K. Gormsen, H. M. Pedersen, and K. L. Gammelmark, "Ultrasound research scanner for real-time synthetic aperture image acquisition," IEEE Trans. Ultrason., Ferroelec., Freq. Contr., vol. 52 (5), pp. 881-891, May 2005.

[14] P. Tortoli, L. Bassi, E. Boni, A. Dallai, F. Guidi, and S. Ricci, "ULAOP: An advanced open platform for ultrasound research," IEEE Trans. Ultrason., Ferroelec., Freq. Contr., vol. 56, no. 10, pp. 2207-2216, Oct. 2009.

[15] J. A. Jensen, H. Holten-Lund, R. T. Nilsson, M. Hansen, U. D. Larsen, R. P. Domsten, B. G. Tomov, M. B. Stuart, S. I. Nikolov, M. J. Pihl, Y. Du, J. H. Rasmussen, and M. F. Rasmussen, "SARUS: A synthetic aperture real-time ultrasound system," IEEE Trans. Ultrason., Ferroelec., Freq. Contr., vol. 60, no. 9, pp. 1838-1852, 2013. 
[16] S. S. Brunke, M. F. Insana, J. J. Dahl, C. Hansen, M. Ashfaq, and H. Ermert, "An ultrasound research interface for a clinical system," IEEE Trans. Ultrason., Ferroelec., Freq. Contr., vol. 54, no. 1, pp. 198-210, January 2007.

[17] H. J. Hewener, H. J. Welsch, C. Günther, H. Fonfara, S. H. Tretbar, and R. M. Lemor, "A highly customizable ultrasound research platform for clinical use with a software architecture for $2 \mathrm{~d}$-/3d-reconstruction and processing including closed-loop control," IFMBE Proceedings, vol. 25, no. 2, pp. 342-345, 2009.

[18] V. Shamdasani, U. Bae, S. Sikdar, Y. M. Yoo, K. Karadayi, R. Managuli, and Y. Kim, "Research interface on a programmable ultrasound scanner," Ultrasonics, vol. 48, no. 3, pp. 159-168, 2008.

[19] M. C. Hemmsen, S. I. Nikolov, M. M. Pedersen, M. J. Pihl, M. S. Enevoldsen, J. M. Hansen, and J. A. Jensen, "Implementation of a versatile research data acquisition system using a commercially available medical ultrasound scanner," IEEE Trans. Ultrason., Ferroelec., Freq. Contr., vol. 59, no. 7, pp. 1487-1499, 2012.

[20] J. A. Jensen, "Safety assessment of advanced imaging sequences, II: simulations," IEEE Trans. Ultrason., Ferroelec., Freq. Contr., p. Submitted, 2015.

[21] Onda, "Methodology for Onda's hydrophone calibrations," Onda Corporation, 592 Weddell Drive, Suite 7, Sunnyvale, CA 94089, USA, Tech. Rep., 2012.

[22] - "AIMS III with Soniq 5.0 Software," Ondo Corporation, 592 Weddell Drive, Suite 7, Sunnyvale, CA 94089, USA, Tech. Rep., 2010.

[23] D. H. Evans and W. N. McDicken, Doppler Ultrasound, Physics, Instrumentation, and Signal Processing. New York: John Wiley \& Sons, 2000.

[24] J. A. Jensen, Estimation of Blood Velocities Using Ultrasound: A Signal Processing Approach. New York: Cambridge University Press, 1996.

[25] J. A. Jensen, A. H. Brandt, and M. B. Nielsen, "Convex array vector velocity imaging using transverse oscillation and its optimization," IEEE Trans. Ultrason., Ferroelec., Freq. Contr., p. Submitted, 2015.

[26] C. A. Villagomez-Hoyos, M. B. Stuart, and J. A. Jensen, "Adaptive multi-lag for synthetic aperture vector flow imaging," in Proc. IEEE Ultrason. Symp., 2014, pp. 1722-1725.

[27] S. G. Foster, "A pulsed ultrasonic flowmeter employing time domain methods," Ph.D. dissertation, Dept. Elec. Eng., University of Illinois, Urbana, Ill., 1985.

[28] O. K. Bates, "Thermal conductivity of liquid silicones," Ind. Eng. Chem., vol. 41, no. 9, pp. 1966-1968, 1949. [Online]. Available: http://dx.doi.org/10.1021/ie50477a030

[29] K. L. Gammelmark and J. A. Jensen, "Multielement synthetic transmit aperture imaging using temporal encoding," IEEE Trans. Med. Imag., vol. 22, no. 4, pp. 552-563, 2003.

\section{BIBLIOGRAPHIES}

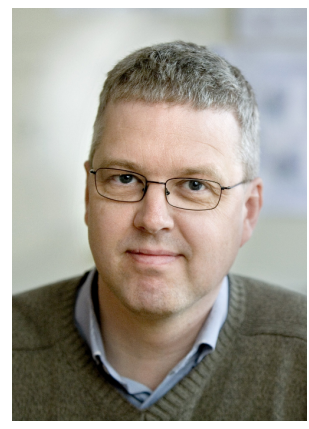

Jørgen Arendt Jensen earned his Master of Science in electrical engineering in 1985 and the $\mathrm{Ph} . \mathrm{D}$. degree in 1989, both from the Technical University of Denmark (DTU). He received the Dr.Techn. degree from the university in 1996. He has published more than 400 journal and conference papers on signal processing and medical ultrasound and the book "Estimation of Blood Velocities Using Ultrasound", Cambridge University Press in 1996. He is also developer of the Field II simulation program. He has been a visiting scientist at Duke University, Stanford University, and the University of Illinois at Urbana-Champaign. $\mathrm{He}$ is currently full professor of Biomedical Signal Processing at DTU, Department of Electrical Engineering and head of Center for Fast Ultrasound Imaging since 1998. He has given courses on blood velocity estimation at both Duke University and University of Illinois and teaches medical imaging at the Technical University of Denmark. He has given several short courses on simulation, synthetic aperture imaging, and flow estimation at international scientific conferences. He has received several awards for his research and is an IEEE Fellow. His research is centered around simulation of ultrasound imaging, synthetic aperture imaging, vector blood flow estimation, and construction of ultrasound research systems.

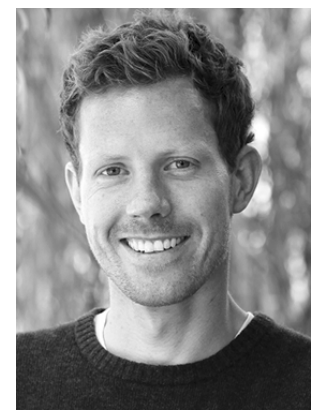

Morten Fischer Rasmussen was born in 1981. He received the B.Sc., M.Sc., and Ph.D. degrees in electrical engineering from the Technical University of Denmark (DTU) in 2008, 2011, and 2014, respectively. In 2007, he spent ten months at the Technische Universitt Berlin, Berlin, Germany, working on the BeeSat satellite. He is currently a postdoctoral research fellow at Stanford University. His current research interests include 3-D ultrasound imaging, synthetic aperture imaging techniques, and ultrasound neurostimulation.

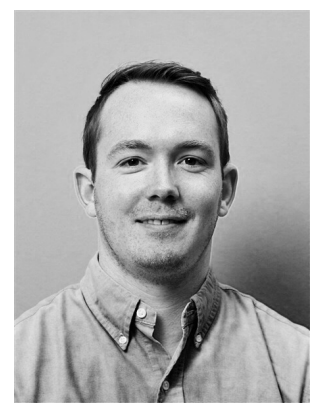

Michael Johannes Pihl, born 1983, received his M.Sc. degree in biomedical engineering in 2009 from the Technical University of Denmark (DTU) and the University of Copenhagen, Denmark. In 2007 he spent seven months at the Biomedical Engineering Department at Duke University, Durham, NC researching ultrasonic clutter. In 2012 he earned his Ph.D. degree in biomedical engineering at the Center for Fast Ultrasound Imaging at DTU. The topic of his degree and postdoctoral work was ultrasonic velocity estimation - in particular the estimation of all three spatial velocity components. His other interests include medical imaging and digital signal processing. Since 2014, he works as an algorithm developer in the R\&D department at Widex - a Danish hearing 
aids manufacturer.

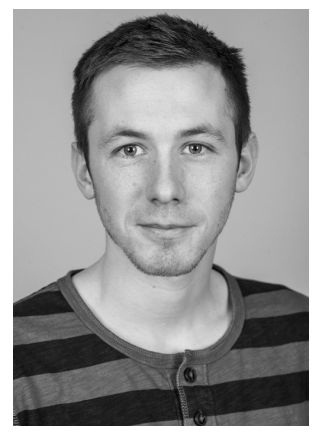

Simon Holbek, born 1986, received his M.Sc. degree in physics in 2013 from the Niels Bohr Institute, University of Copenhagen, Denmark. He is currently pursuing his Ph.d. degree in electrical and biomedical engineering at the center for Fast Ultrasound Imaging at DTU. The topics of his Ph.d. thesis is estimation of 3-D vector flow.

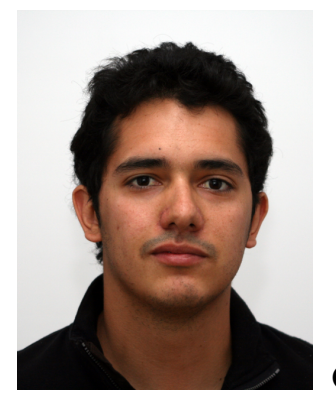

Carlos Armando Villagómez Hoyos was in born 1985. He received his B.Sc. in electronic engineering during 2008, and M.Sc. degree in digital signal processing in January 2013 both from the National Autonomous University of Mexico. He spent six months at the Ultrasound Laboratory at the Federal University of Rio de Janiero in 2012. $\mathrm{He}$ is now pursuing his $\mathrm{PhD}$ degree in biomedical engineering at the Center for Fast Ultrasound Imaging at the Technical University of Denmark. The topic of his $\mathrm{PhD}$ research is "Optimal synthetic aperture duplex imaging".

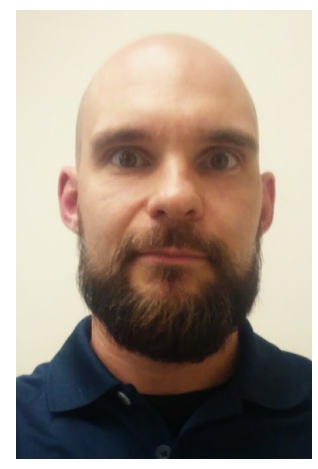

David P. Bradway earned his Ph.D. degree in biomedical engineering in 2013 from Duke University. Afterward, he was a guest postdoc at the Technical University of Denmark (DTU), supported by a Whitaker International Program Scholarship. He has conducted research internships at the Cleveland Clinic Foundation, Volcano Corporation, and Siemens Healthcare. He is currently a research scientist in the Biomedical Engineering Department at Duke University. His research interests include ultrasonic elasticity imaging, synthetic aperture imaging, spatial coherence, com- putational processing, and transverse flow imaging methods.

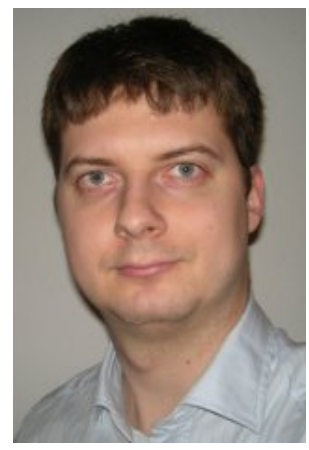

Matthias Bo Stuart received the M.Sc. and Ph.D. degrees in Computer Engineering in 2006 and 2010 respectively, both from the Technical University of Denmark, Lyngby, Denmark. He is currently a postdoc with the Center for Fast Ultrasound Imaging at the Department of Electrical Engineering, Technical University of Denmark. His research interests include synthetic aperture methods for both anatomical and flow imaging, ultrasound systems, and realtime implementations of ultrasound processing algorithms.

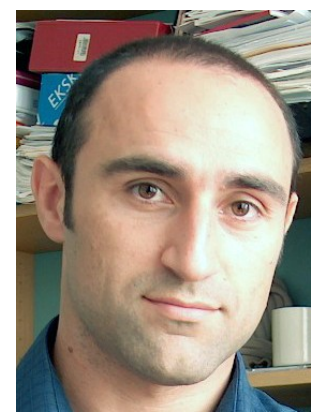

Borislav Gueorguiev Tomov obtained his M. Sc. degree in Electronics Engineering at the Technical University of Sofia, Bulgaria, in 1996, and his Ph.D. degree in Medical Electronics at the Technical University of Denmark in 2003. His research interests include medical ultrasound signal processing, and ultrasound scanner architectures and implementations. 